\title{
DA SISTEMÁTICA À CONSERVACÃ̃O DE PEIXES NEOTROPICAIS: O LEGADO CIENTÍFICO DO PROFESSOR DR. RICARDO DE SOUZA ROSA
}

\author{
FROM SYSTEMATICS TO CONSERVATION OF NEOTROPICAL FISHES: \\ THE SCIENTIFIC LEGACY OF PROFESSOR DR. RICARDO DE SOUZA ROSA \\ DE LA SISTEMÁTICA A LA CONSERVACIÓN DE PECES NEOTROPICALES: \\ EL LEGADO CIENTÍFICO DEL PROFESOR DOCTOR RICARDO DE SOUZA ROSA
}

\author{
Sarah TFL Viana ${ }^{1}$ \\ Bráulio Almeida Santos ${ }^{2}$ \\ Robson Tamar da Costa Ramos ${ }^{3}$
}

\section{Resumo}

A ictiologia neotropical caracteriza-se por uma área da Biologia bem consolidada no Brasil, país com a mais rica biodiversidade do planeta. Para tanto, esforços de pesquisa e dedicação de inúmeros cientistas foram imprescindíveis em âmbitos nacional e internacional. O Professor Dr. Ricardo Rosa da Universidade Federal da Paraíba, consagrado ictiólogo e militante ambiental, se destaca como produtor de conhecimento científico neste parâmetro, principalmente devido ao seu pioneirismo em sistemática de peixes e conservação da biota aquática na América do Sul. Recentemente aposentado, Ricardo Rosa deixou um legado científico expressivo especialmente no Nordeste brasileiro onde atuou por mais de 40 anos na formação básica oferecida na sala de aula ao alunos de graduação à formação de pesquisadores de alto nível durante sua supervisão na pós-graduação. A fim de homenagear este cientista ímpar iremos relatar aqui a sua trajetória profissional.

Palavras-chave: ictiologia; docência; pesquisa; Nordeste brasileiro.

\section{Resumen}

La ictiología es un área de la Biología bien consolidada en Brasil, país con la más rica biodiversidad del mundo. Para ello, los esfuerzos de investigación y la dedicación de inúmeros investigadores han sido esenciales a nivel nacional e internacional. El Profesor Doctor Ricardo Rosa de la Universidad Federal de Paraíba, consagrado ictiólogo y militante ambiental, se ha destacado como importante productor de conocimiento científico, principalmente debido a su pionerismo en la sistemática

1 South African Institute for Aquatic Biodiversity, Private Bag 1015, Grahamstown, 6140, South Africa. Email: stviana@gmail.com

2 Departamento de Sistemática e Ecologia, CCEN, Universidade Federal da Paraíba, Campus Universitário I, CEP 58051-90o, João Pessoa, PB, Brazil.

3 Departamento de Sistemática e Ecologia, CCEN, Universidade Federal da Paraíba, Campus Universitário I, CEP 58051-900, João Pessoa, PB, Brazil. 
de peces y conservación de la biota acuática de Sudamérica. Recientemente retirado, Ricardo Rosa ha dejado un legado científico expresivo especialmente en el Noreste de Brasil, donde actuó por más de 40 años en la formación básica de estudiantes de pregrado y en la formación de investigadores de alto nivel en el posgrado. Para homenajear este científico impar relatamos aquí su trayectoria profesional.

Palabras-clave: ictiología; enseñanza; investigacion; Nordeste brasileño.

\begin{abstract}
Neotropical ichthyology comprises a well consolidated area within Biology in Brazil, a country with the world's richest biodiversity. Research efforts and academic commitment of a number of scientists were indispensable in the national and international scopes. Professor Dr. Ricardo Rosa from the Federal University of Paraíba, devoted ichthyologist and environmental activist, stands out for its scientific productivity due its pioneer achievements in fish systematics and conservation of the aquatic biota in South America. Recently retired, Ricardo Rosa left behind an expressive scientific legacy especially in the Brazilian Northeast region where he was based for over 40 years. Whilst at UFPB, he acted passionately in the formation of undergraduate students during his lectures and new generation of high academic standards researchers while supervising them in the local post-graduation programs. In order to honor this outstanding scientist, we will report here his professional pathway.
\end{abstract}

Keywords: ichthyology; lecturing; research; Brazilian Northeast.

\title{
INTRODUÇÃO
}

A ictiologia brasileira constitui uma área da Biologia cuja base científica e educacional se estabeleceu desde a chegada da família real Portuguesa ao país, no século XVI. O alicerce ictiológico, contudo, foi instituído quando da vinda da imperatriz Maria Leopoldina da Áustria, primeira esposa do imperador de Portugal, D. Pedro I, ao Brasil, em 1817. A imperatriz era, sem dúvida, uma mulher politicamente engajada, sendo por muitos considerada a primeira mulher chefe de Estado do Brasil independente, além de ser excepcionalmente culta, e cuja exigência primordial foi ser acompanhada por uma comitiva de cientistas bávaros como parte da sua missão ao Novo Mundo, entre eles o primeiro especialista atuante no país, Johann Baptist von Spix. Ictiólogos brasileiros e/ou brasileiros naturalizados tiveram maior progressão em suas pesquisas a partir do século XX como, por exemplo, Alípio de Miranda-Ribeiro do Museu Nacional do Rio de Janeiro, Hermann Friedrich Albrecht von Ihering e Antônia de Amaral Campos ambos do Museu Paulista, Emílio Augusto Goeldi do Museu Paraense, e Elisabeth Maria Santana Honda do Instituto Nacional de Pesquisas Amazônicas. 
No Nordeste do Brasil, o professor Dr. Ricardo de Souza Rosa da Universidade Federal da Paraíba (UFPB), fundador e chefe do Laboratório de Ictiologia do Departamento de Sistemática e Ecologia, atuou como professor e pesquisador ao longo de mais de 40 anos, tendo prestado uma contribuição destacada ao desenvolvimento da Ictiologia Neotropical, com produtividade que se estende à sistemática, ecologia e conservação de peixes cartilaginosos, em especial, e aos peixes ósseos marinhos. Ricardo Rosa se dedicou à formação da vida acadêmica de centenas de estudantes e ictiólogos brasileiros. Temos, portanto, o prazer em homenagear este consagrado cientista e ativista ambiental recentemente aposentado ao relatar aqui a sua trajetória profissional.

\section{BIOGRAFIA}

Ricardo de Souza Rosa nasceu na cidade de Porto Alegre, no Estado do Rio Grande do Sul, em 27 de abril de 1954. Filho de David de Souza Rosa e Refcá Canetti Rosa, Ricardo Rosa era um curioso e admirador da natureza desde sua infância, mais particularmente devido à influência da uma tia materna e da sua irmã mais velha que o estimularam na direção da Biologia Marinha. Ricardo morou em Porto Alegre até os oito anos de idade, tendo-se mudado com sua família para o Rio de Janeiro; em 1969, mudou-se para a cidade de São Paulo. Nesta cidade, Ricardo estudou o Ensino Médio e, já naquele tempo, seu interesse pela zoologia se manifestava - era frequentador assíduo do Museu de Zoologia da Universidade de São Paulo (MZUSP), à época denominado Departamento de Zoologia da Secretaria de Agricultura de São Paulo, e das bases de apoio do Instituto Oceanográfico e do Instituto de Biologia Marinha da Universidade de São Paulo, situados no litoral paulista. Neste último, obteve seu primeiro certificado em Biologia Marinha, com a participação em um curso de extensão ministrado pelo Professor Paulo Sawaya, em 1970. Cursou Bacharelado em Ciências Biológicas na Universidade de São Paulo entre os anos de 1972 a 1976, e quando estagiou na Seção de Peixes do MZUSP, a partir de 1973, sob a supervisão dos Professores Drs. José Lima de Figueiredo e Heraldo Britski, renomados especialistas da área, e então curadores da coleção de Ictiologia do MZUSP. Logo após a finalização da graduação, sob indicação de seus professores, ingressou no Setor de Peixes do Instituto de Pesquisas da Marinha, situado em Arraial do Cabo, Rio de Janeiro, para a realização de um estágio remunerado. A permanência ali foi curta uma vez que a Universidade Federal da Paraíba vivia no final da década de 1970 uma importante expansão sob a gestão do Reitor, Lynaldo Cavalcanti de Albuquerque. A estratégia do Reitor era buscar no país e fora dele nomes jovens e destacados para dar início a uma nova linha de ação da Universidade, inclusive no momento em que estava sendo criado o curso de Biologia, e Ricardo foi um dos nomes escolhidos, tendo apenas finalizado sua graduação. Dessa forma, em 1977, assumiu a vaga de Professor Colaborador do Departamento de Sistemática e Ecologia da UFPB e logo iniciou sua pesquisa sobre 
os peixes da região. Como era o plano da UFPB e parte do projeto de expansão das universidades no país, Ricardo obteve financiamento do programa PICD/CAPES, para realizar seus estudos de mestrado no Virginia Institute of Marine Science, College of William and Mary, nos Estados Unidos, sob orientação do renomado especialista americano em elasmobrânquios, Dr. John A. Musick. Uma avaliação prévia no final do seu período de realização da dissertação de mestrado, dada a sua extensão, levou-o a ter seu projeto indicado para o nível de Doutorado. No entanto, Ricardo precisou voltar ao Brasil, visto que terminava seu período de afastamento e financiamento para permanência no exterior. Simultaneamente ao retorno às atividades da UFPB, Ricardo produziu os últimos investimentos sobre seu trabalho e concluiu sua tese de doutorado em 1985. Em 1989, afastou-se da Universidade para a realização do pós-doutorado na Universidade de Alberta, Canadá, sob a supervisão do Dr. Joseph S. Nelson.

Paralelamente à sua atuação como professor, Ricardo foi um pesquisador atuante na produção de conhecimentos em campos como a taxonomia, ecologia e conservação de peixes, com ênfase em elasmobrânquios, e teve uma destacada atuação fora dos muros da Universidade, como a participação na Sociedade Brasileira de Ictiologia e na Sociedade Brasileira para o Estudo de Elasmobrânquios, das quais foi sócio-fundador, respectivamente, em 1981 e 1995. Na SBEEL, foi presidente eleito por três períodos entre 2000 e 2016, e é o tesoureiro da gestão atual. Foi também peça importante na avaliação do estado de ameaça a extinção das espécies de peixes neotropicais realizado no país. Ao longo desta jornada, Ricardo casou-se duas vezes, teve três filhos e, mais recentemente, tornou-se avô de um menino.

\section{DOCÊNCIA NO NORDESTE DO BRASIL}

Ricardo Rosa contribuiu, no início de sua atuação como professor da UFPB, no curso de Licenciatura em Educação Física e no extinto curso de Licenciatura em Ciências, ministrando a disciplina Biologia Geral. No curso de graduação em Ciências Biológicas, seu principal foco de atuação, ministrou as disciplinas Zoologia dos Vertebrados (peixes e tetrápodes), Metazoários Deuterostomados (Vertebrados e invertebrados parentes), Anatomia Comparada de Vertebrados, Fundamentos de Sistemática e Biogeografia, e disciplinas de cunho geral como 'Temas Atuais em Biologia II', seja como único ministrante ou em colaboração com outros professores do departamento. Cedo em sua carreira, criou e ministrou as disciplinas optativas Ictiologia e Biologia dos Elasmobrânquios, para despertar o interesse de estudantes na área de peixes. Mais recentemente, participou do curso de Ensino à Distância de Licenciatura em Ciências Biológicas, onde ministrou as disciplinas Fundamentos de Sistemática e Biogeografia e Zoologia dos Vertebrados.

No Programa de Pós-graduação em Ciências Biológicas da UFPB (PPGCB) com área de concentração em Zoologia e criado em 1980, exerceu papel fundamental 
na estruturação e fortalecimento do programa. A partir de 1985, ingressou neste programa e ministrou as disciplinas de Ictiologia, Métodos de Coleta e Preparação de Vertebrados e Invertebrados, Teorias e Métodos em Zoologia Sistemática, Biologia Comparada, Introdução à Zoologia de Campo, bem como criou a disciplina optativa Conservação da Biodiversidade. Também ingressou em mais dois programas de pós-graduação da UFPB - Desenvolvimento e Meio Ambiente (PRODEMA) e Ecologia e Monitoramento Ambiental (PPGEMA), nos quais assumiu as disciplinas Conservação e Gestão de Recursos Naturais, Biologia da Conservação da Fauna e da Flora e Biologia da Conservação, em colaboração com outros professores desses Programas. Ao todo, Ricardo Rosa orientou 26 alunos de graduação, 12 estudantes de iniciação científica, um estudante de especialização, e 53 alunos de pós-graduação em nível de Mestrado (42) e Doutorado (11), além de quatro co-orientações nos dois níveis da pós-graduação, desempenhando grande impacto na formação da nova geração de docentes e pesquisadores em Ictiologia e Conservação Animal atuantes no Brasil e no exterior. Participou de 155 bancas de avaliação de conclusão de cursos de pós-graduação, 49 de graduação, 28 qualificações, e 12 concursos públicos para provimento de cargo de professor do magistério superior. Concomitante às atividades de docência, Ricardo Rosa atuou ativamente na administração da UFPB como coordenador e vice-coordenador do PPGCB (quatro períodos entre 1985-200o), chefe e sub-chefe do Departamento de Sistemática e Ecologia (três períodos entre 1992-2011) e vice-coordenador do Núcleo de Estudos e Pesquisa dos Recursos do Mar (NEPREMAR, entre 1988-1989).

\section{PIONEIRISMO EM ICTIOLOGIA NEOTROPICAL}

O interesse e a atuação científica sobre a diversidade de peixes neotropicais renderam a Ricardo Rosa o título de ictiólogo pioneiro em estudos sobre sistemática de raias de água doce, fato embasado na sua tese intitulada "Uma revisão Sistemática das raias de água doce da América do Sul (Chondrichthyes: Potamotrygonidae)". O trabalho inclui a redescrição de 19 espécies nominais, levantamento de caracteres morfológicos ainda desconhecidos, discussão das relações filogenéticas internas do grupo através de análises cladística e biogeográfica, e o reconhecimento de três gêneros na composição da família Potamotrygonidae, e resultou na descrição de um gênero e sua espécie-tipo, Plesiotrygon iwamae Rosa, Castello \& Thorson, 1987.

Ricardo Rosa publicou 68 artigos em periódicos científicos nacionais e internacionais, entre eles seus primeiros artigos de autoria única "A natação dos peixes" (1978) e "Lista Sistemática de peixes marinhos da costa da Paraíba" (1980), na presente revista. Em sistemática ictiológica, destacam-se Rosa (1991) e Rosa e Gadig (2014) e a descrição de espécies novas como, por exemplo, barrigudinhos em Rosa e Costa (1993) além de raias marinhas e dulcícolas em Gomes et al. (2000), Rosa et al. (2008), e Carvalho et al. (2016). Outro passo importante consistiu em 
publicações sobre a diversidade de peixes marinhos do Nordeste brasileiro, até então pouco conhecida, como Rosa et al. (1997), Rocha et al. (1998), Rocha e Rosa (2001), e Souza et al. (2007). Vários trabalhos foram realizados por alunos sob sua orientação e posteriormente publicados, abrangendo a biologia reprodutiva e alimentar de espécies nativas de elasmobrânquios (e.g. Shibuya et al. 2005; Moro et al. 2012; Márquez-Velásquez et al. 2019). Aspectos da pesca artesanal da costa da Paraíba também foram objetos de estudo (e.g. Dias et al. 2007; Mariano e Rosa 2012; Paulo Júnior et al. 2012; Nascimento et al. 2016), indicando o envolvimento de Ricardo Rosa na articulação político-científica entre as comunidades pesqueiras e autoridades locais para minimizar os impactos de pesca na região e garantir a sustentabilidade dos estoques pesqueiros. Sobre a fauna recifal, atuou como colaborador em estudos ictiológicos regionais, mais particularmente relacionados à costa Nordeste do país sobre estrutura de comunidades (e.g. Ilarri et al. 2017), zoogeografia (e.g. Feitoza et al. 2005; Pinheiro et al. 2018), uso de habitat (e.g. Aguiar et al. 2009; Gama e Rosa 2015; Medeiros et al. 2016) e tamanho populacional (Castro e Rosa 2005; Freitas et al. 2009). Quando se analisa o Currículo Lattes de Ricardo Rosa, se tem o registro de apenas um artigo que versa sobre os peixes de água doce da Caatinga. No entanto, perspicaz que sempre foi, sua atuação no estímulo aos estudos da Caatinga foi central. Ele foi convidado para compor o grupo de pesquisadores que estabeleceu, a pedido do Ministério do Meio Ambiente, as áreas prioritárias para a conservação da Caatinga. A partir dessa experiência, coordenou um esforço que resultou na publicação do capítulo de livro "Diversidade, padrões de distribuição e conservação dos peixes da Caatinga”, Rosa et al. (2003), que se tornou um clássico da literatura, nesse campo, e que é citação frequente daqueles que trabalham com a ictiofauna da Caatinga. Também participou da atualização dos dados dos peixes da região, recentemente publicado em Lima et al. (2017).

Uma vasta contribuição no formato de livros e capítulos de livros está registrada em seu currículo. Participou como organizador em três livros, como autor único em cinco capítulos de livros e como coautor em 170 capítulos, publicações maioritariamente relacionadas à diversidade de elasmobrânquios ocorrentes na América do Sul. O livro "Rayas de agua dulce (Potamotrygonidae) de Suramérica” recentemente publicado em duas edições (Lasso et al. 2013, 2016) é destacado devido à minuciosa apresentação de dados taxonômicos, ecológicos e de esforços sobre conservação regionais do grupo. Publicações importantes na mesma área são o histórico do conhecimento e avanços de pesquisas sobre biologia de potamotrigonídeos (Rosa et al. 2010). Outros estudos relacionados à sistemática de peixes foram publicados em Rosa e Carvalho (2002), Carvalho et al. (2003), e Rosa e Carvalho (2007). No presente ano, Ricardo Rosa atuou como colaborador em uma publicação sobre aspectos dos ecossistemas de corais mesofóticos do Brasil, evidenciando sua continua ação como pesquisador ao longo de toda a carreira.

Outro ponto de destaque dentro da carreira profissional de Ricardo Rosa é a fundação da Coleção Ictiológica da Universidade Federal da Paraíba (CI-UFPB), 
em 1977, com a colaboração das Professoras Graciela Cannella e Maria Margarida Rodrigues. Ricardo, com seus alunos, foi o principal promotor do acervo de peixes ósseos e cartilaginosos marinhos e estuarinos da CI-UFPB, além do acervo de raias de água doce da América do Sul, grupo do qual é especialista. A partir dos anos 2000, a coleção recebeu a contribuição do Prof. Robson T. C. Ramos (seu ex-aluno, professor do mesmo Departamento, a partir de 1990) e sua equipe, em especial seu ex-aluno Dr. Telton P. A. Ramos, resultando numa coleção importante de peixes continentais do Nordeste. Atualmente, a coleção apresenta um acervo de quase de 12.000 lotes de peixes ósseos e cartilaginosos, além de feiticeiras e lampréias, somando mais de 175.000 espécimes (Carvalho-Rocha et al. 2019). A coleção representa hoje um importante acervo da ictiofauna marinha da Paraíba e de outros Estados do Nordeste do Brasil, e é a mais representativa coleção da ictiofauna dulcícola da porção norte da Caatinga brasileira, com amostragens entre os Estados do Maranhão e Alagoas, e da Mata Atlântica entre os Estados de Rio Grande do Norte e Alagoas.

\section{CONSERVAÇÃO DA FAUNA ICTIOLÓGICA BRASILEIRA}

Sendo um amante da natureza e militante ambiental, Ricardo Rosa engajouse em garantir efetivamente a conservação da fauna ictiológica brasileira. Ainda no início de suas atividades acadêmicas, elaborou a primeira lista de espécies de peixes ameaçadas de extinção do Brasil, em 1990, o que rendeu um prêmio de honra ao mérito da Sociedade Brasileira de Zoologia (a lista foi formalmente publicada em Rosa e Menezes 1996). A partir de então, passou a integrar importantes iniciativas do gênero no país e em âmbito internacional, como a Lista Vermelha de Espécies Ameaçadas da International Union for Conservation of Nature (IUCN) em 2004, o Plano Nacional de Ação para a Conservação e Manejo de estoque de peixes elasmobrânquios no Brasil (Sociedade Brasileira para o Estudo de Elasmobrânquios (SBEEL) em 2005, e o Livro Vermelho da Fauna Brasileira Ameaçada de Extinção (ICMBio/MMA) em 2008 e 2018. Atuou também nas ações de estabelecimento de áreas naturais protegidas no Estado da Paraíba, como Picãozinho, Areia Vermelha e a Área de Proteção Ambiental Naufrágio Queimado (a maior do gênero no Estado, com aproximadamente $422 \mathrm{~km}^{2}$ de extensão). Seu rigor técnico e capacidade de diálogo com o setor público foram decisivos para a agenda de conservação marinha do Estado da Paraíba.

\section{CONCLUSÃO}

O que se pode depreender nas linhas que traçamos acima permite uma leitura panorâmica do profissional multitarefa e perspicaz que tem sido Ricardo Rosa ao longo de seus anos de contribuição como acadêmico da UFPB. Seu amplo leque de atuação se estende desde a formação básica oferecida na sala de aula aos alunos de graduação 
à formação de pesquisadores de alto nível para os quais foi inspiração e suporte ao longo de sua carreira, assim como à atuação dele próprio como destacado produtor de conhecimento científico, nos âmbitos nacional e internacional, em especial, na América Latina. Da mesma forma, podemos observar como se constituiu em um profissional com os olhos voltados para a sociedade, enquanto dispôs sua formação e experiência para realizar com efetividade seu serviço ao país, em especial no trabalho árduo de conservação da vida na nação com a mais rica biodiversidade do planeta. O relato acima nos remete à decisão acertada dos gestores que garimparam o país e outros pontos da América do Sul em busca de nomes para constituir o destacado grupo de profissionais que deram o passo inicial para a formação do Curso de Biologia da UFPB, que se projetou como um grupo importante na área de ciências naturais de nossa nação, e que tem em Ricardo Rosa um de seus nomes mais importantes.

\section{AGRADECIMENTOS}

Os autores agradecem a Revista Nordestina de Biologia em especial ao Editorchefe, Dr. Reinaldo FP de Lucena, por conceder esta oportunidade em homenagear o Prof. Ricardo Rosa. Ao Programa de Pós-Graduação em Ciências Biológicas e ao Departamento de Sistemática e Ecologia da Universidade Federal da Paraíba, sinceros agradecimentos pelo apoio acadêmico e científico.

\section{LITERATURA CITADA}

Aguiar AA, Valentin JL, Rosa RS. 2009. Habitat use by Dasyatis americana in a southwestern Atlantic oceanic island. Journal of the Marine Biological Association of the United Kingdom, 89:1147-1152. https://doi.org/10.1017/Soo25315409000058

Carvalho MR, Lovejoy NR, Rosa RS. 2003. Family Potamotrygonidae (River stingrays). In: Reis RE, Kullander SO, Ferraris Jr. CJ (Eds), Check List of the Freshwater Fishes of South and Central America, Porto Alegre: Edipucrs, p. 22-28.

Carvalho MR, Rosa RS, Araújo MLG. 2016. A new species of Neotropical freshwater stingray (Chondrichthyes: Potamotrygonidae) from the Rio Negro, Amazonas, Brazil: the smallest species of Potamotrygon. Zootaxa, 4107(4):566. http://dx.doi. org/10.11646/zootaxa.4107.4.5

Carvalho-Rocha YGP, Beltrão GBM, Cortez SC, Sales J, Figueiredo-Filho JM, Freitas NDA, Ramos TPA, Oliveira-Silva L, Ramos RTC, Rosa RS. 2019. Forty years of the Ichthyological Collection of Universidade Federal da Paraíba. Boletim Sociedade Brasileira de Ictiologia, 129:137-139. 
Castro ALF, Rosa RS. 2005. Use of natural marks on population estimates of the nurse shark, Ginglymostoma cirratum, at Atol das Rocas Biological Reserve, Brazil. Environmental Biology of Fishes, 72(2):213-221. https://doi.org/10.1007/s10641-0041479-7

Dias TLP, Rosa RS, Damasceno LCP. 2007. Aspectos socioeconômicos, percepção ambiental e perspectivas das mulheres marisqueiras da Reserva de Desenvolvimento Sustentável Ponta do Tubarão (Rio Grande do Norte, Brasil). Gaia Scientia, 1(1):25-35.

Feitoza BM, Rosa RS, Rocha Filho LA. Ecology and zoogeography of deep-reef fishes in northeastern Brazil. Bulletin of Marine Science,76(3):725-742.

Freitas RHA, Rosa RS, Wetherbee BM, Gruber SH. 2009. Population size and survivorship for juvenile lemon sharks, Negaprion brevirostris, on their nursery grounds at a marine protected area in Brazil. Neotropical Ichthyology, 7:205-212. http://dx.doi.org/10.159o/S1679-62252009000200011

Gama C, Rosa RS. 2015. Uso de recursos e dieta das raias deágua doce (Chondrichthyes, Potamotrygonidae) da Reserva Biológica do Parazinho. Biota Amazônia, 5(1):90-98. http://dx.doi.org/10.18561/2179-5746/biotaamazonia.v5nıp9o-98

Gomes UL, Rosa RS, Gadig OBF. 2000. Dasyatis macrophthalma sp. n.: a new species of stingray (Chondrichthyes: Dasyatidae) from the Southwestern Atlantic. Copeia, 2:510-515.

Ilarri MI, Souza AT, Rosa RS. 2017. Community structure of reef fishes in shallow waters of the Fernando de Noronha archipelago: effects of different levels of environmental protection. Marine and Freshwater Research, 68:1303-1316. https://doi.org/10.1071/ MF16071

Lasso CA, Rosa RS, Morales-Betancourt M, Garrone-Neto D, Carvalho MR. 2016. Rayas de agua dulce (Potamotrygonidae) de Suramérica. Parte II: Colombia, Brasil, Perú, Bolivia, Paraguay, Uruguay y Argentina, 1st ed., Bogotá: Instituto de Investigación de Recursos Biológicos Alexander von Humboldt, 435 p.

Lasso CA, Rosa RS, Sanchez-Duarte P, Morales-Betancourt M, Agudelo-Cordoba E. 2013. Rayas de agua dulce (Potamotrygonidae) de Suramérica. Parte I. Colombia, Venezuela, Ecuador, Perú, Brasil, Guyana, Surinam y Guayana Francesa: diversidad, bioecología, uso y conservación, 1st ed., Bogotá: Instituto de Investigación de Recursos Biológicos Alexander von Humboldt, 368 p. 
Lima SMQ, Ramos TPA, Silva MJ, Rosa RS. 2017. Diversity, Distribution, and Conservation of the Caatinga Fishes: Advances and Challenges. In: Silva JMC, Leal IR, Tabarelli M. (Eds), Caatinga, Springer, p. 97-131.

Mariano EF, Rosa RS. 2012. Caracterização da pesca artesanal no litoral da Paraíba: embarcações, procedimentos e capturas da pesca embarcada. Boletim Técnico Científico do CEPENE, 18:61-73.

Medeiros PM, Medeiros AMA, Rosa RS. 2016. Substrate association and patterns of microhabitat use by fishes at Fernando de Noronha. Tropical Oceanography, 44:158171. https://doi.org/10.5914/tropocean.v44i2.8299

Moro G, Charvet P, Rosa RS. 2012. Insectivory in Potamotrygon signata (Chondrichthyes: Potamotrygonidae), an endemic freshwater stingray from the Parnaíba River basin, northeastern Brazil. Brazilian Journal of Biology, 72:885-891. http://dx.doi. org/10.1590/S1519-69842012000500015

Nascimento GCC, Córdula EBL, Lucena RFP, Rosa RS, MourãoJS. 2016. Characterization of artisanal fishing in fish weirs, the north coast of Paraiba, Brazil. Arquivos de Ciências do Mar, 49:92-103. https://doi.org/10.3236o/acmar.v49i2.6585

Paulo Júnior EPN, Xavier JHA, Sassi R, Rosa RS. 2012. Gestão da pesca artesanal na Costa da Paraíba, Brasil: uma abordagem utilizando o Processo Analítico Hierárquico. Revista da Gestão Costeira Integrada, 12:509-520.

Pinheiro HT, Rocha LA, Macieira RM, Carvalho-Filho A, Anderson AB, Bender MG, Di Dario F, Ferreira CEL, Figueiredo-Filho J, Francini-Filho R, Gasparini JL, Joyeux JC, Luiz OJ, Mincarone MM, Moura RL, Nunes JACC, Quimbayo JP, Rosa RS, Sampaio CLS, Sazima I, Simon T, Vila-Nova DA, Floeter SR. 2018. South-western Atlantic reef fishes: Zoogeographical patterns and ecological drivers reveal a secondary biodiversity centre in the Atlantic Ocean. Diversity and Distributions, 24(7):951-965. https://doi. org/10.1111/ddi.12729

Rocha LA, Rosa IML, Rosa RS. 1998. Peixes recifais da costa da Paraíba, Brasil. Revista Brasileira de Zoologia,15:553-566. http://dx.doi.org/10.159o/So101-81751998000200017

Rocha LA, Rosa RS. 2001. Halichoeres brasiliensis (Bloch, 1791), a valid wrasse species (Teleostei: Labridae) from Brazil, with notes on the Caribbean species Halichoeres radiatus (Linnaeus, 1758). Aqua (Neu-Isenburg) Graffignana, 4(3):161-166.

Rosa RS. 1978. A Natação nos peixes. Revista Nordestina de Biologia, 1 (1):149-161. 
Rosa RS. 1980. Lista sistemática de peixes marinhos da Paraíba. Revista Nordestina de Biologia, 3(2):205-226.

Rosa RS. 1991. Paratrygon aiereba (Muller And Henle, 1841) the senior synonym of the freshwaterstingrayDisceus thayeriGarman,1913 (Chondrichthyes:Potamotrygonidae). Revista Brasileira de Zoologia, 7(4):425-437.

Rosa RS, Carvalho MR. 2002. Potamotrygonidae. In: Buckup PA, Menezes NA (Eds), Catálogo dos peixes marinhos e de água doce do Brasil, Rio de Janeiro: Universidade Federal do Rio de Janeiro, Museu Nacional do Rio de Janeiro.

Rosa RS, Carvalho MR. 2007. Família Potamotrygonidae. In: Buckup PA, Menezes NA, Ghazzi MS (Eds). Catálogo das espécies de peixes de água doce do Brasil, Rio de Janeiro: Museu Nacional, p.17-18.

Rosa RS, Carvalho MR, Wanderley CA. 2008. Potamotrygon boesemani (Chondrichthyes: Myliobatiformes: Potamotrygonidae), a new species of Neotropical freshwater stingray from Surinam. Neotropical Ichthyology, 6:1-8. http://dx.doi. org/10.159o/S1679-62252008000100001

Rosa RS, Costa WJEM. 1993. Systematic Revision of the genus Cnesterodon (Cyprinodontiformes: Poeciliidae), with the description of two new species from Brazil. Copeia, 3:696-708. http://dx.doi.org/ 10.2307/1447230

Rosa RS, Charvert-Almeida P, Quijada CCD. 2010. Biology of the South American Potamotrygonid Stingrays. In: Carrier JC, Musick JA, Heithaus MR (Eds), Sharks and their relatives II: biodiversity, adaptive physiology, and conservation, Boca Raton, Florida: CRC Press, p. 241-281.

Rosa RS, Gadig OBF. 2014. Conhecimento da diversidade dos Chondrichthyes marinhos no Brasil: a contribuição de José Lima de Figueiredo. Arquivos de Zoologia, 45:89-104. https://doi.org/10.116o6/issn.2176-7793.v45iespp89-104

Rosa RS, Menezes NA. 1996. Relação preliminar das espécies de peixes (Pisces, Elasmobranchii, Actinopterygii) ameacadas no Brasil. Revista Brasileira de Zoologia, 13(3):647-667. http://dx.doi.org/10.1590/So101-81751996000300015.

Rosa RS, Rosa IML, Rocha LA. 1997. Diversidade da ictiofauna de poças de maré da Praia do Cabo Branco, João Pessoa, Paraíba, Brasil. Revista Brasileira de Zoologia, 14(1):201-212. http://dx.doi.org/10.1590/So101-81751997000100019 
Shibuya A, Rosa RS, Soares MC. 2005. Note on the diet of the guitarfish Rhinobatos percellens (Walbaum, 1792) (Elasmobranchii: Rhinobatidae) from the coast of Paraíba, Brazil. Acta Biologica Leopoldensia, São Leopoldo, 27(1):63-64.

Souza AT, Ilarri MI, Medeiros PM, Grempel RG, Rosa RS, Sampaio CLS. 2007. Fishes (Elasmobranchii and Actinopterygii) of Picãozinho reef, Northeastern. Zootaxa, 1608:11-19. http://dx.doi.org/10.11646/zootaxa.1608.1.2

Velásquez VM, Rosa RS, Galindo E, Navia AF. 2019. Feeding habits and ecological role of the freshwater stingray Potamotrygon magdalenae (Elasmobranchii: Batoidea), combining gut-content and stable isotope analysis. Environmental Biology of Fishes, 102(8):1119-1136. https://doi.org/10.1007/s10641-019-00897-o 